\title{
Focal takotsubo syndrome triggered by adrenal crisis following STEMI: case report.
}

\author{
Andrea Falcetta ${ }^{1}$, Eleonora Bonfanti ${ }^{2}$, Roberta Rossini $^{1}$, and Giuseppe Lauria ${ }^{1}$ \\ ${ }^{1} \mathrm{~S}$ Croce and Carle Cuneo Hospital Districts \\ ${ }^{2}$ Azienda Ospedaliero Universitaria Città della Salute e della Scienza di Torino
}

March 7, 2022

\begin{abstract}
Differential diagnosis of shock occurring within few hours to percutaneous coronary intervention (PCI) performed to treat acute ST-segment elevation myocardial infarction (STEMI) encompass periprocedural complications and causes of other type, including adrenal crisis (AC), sometimes presenting as Takotsubo syndrome.
\end{abstract}

\section{INTRODUCTION}

Percutaneous Coronary Intervention (PCI) is currently indicated for the treatment of ST-segment elevation myocardial infarction (STEMI) (1), nevertheless, in about $1 \%$ of cases, PCI-related complications may occur leading to shock and early mortality (2). Roughly, these ones can be divided among coronary artery (3), vascular (4) and of other type (including bleeding and infection) and may present with ST-segment elevation (STE). Alongside the latter, also early post-acute myocardial infarction pericarditis (5) and takotsubo syndrome (TTS) (6) may show STE and should be included in differential diagnosis. Moreover it is also know that the apical apical form of TTS is the most frequent one and that it may be triggered by an adrenal crisis (AC) (7) which, in turn, can be a consequence of a myocardial infarction and that may present with severe hypotension or shock, electrolyte disturbances, prerenal failure and reduced peripheral vascular resistances (8)(9) making the diagnosis, especially after STEMI, a real challenge.

\section{CASE PRESENTATION}

A 74-year-old caucasian woman, diagnosed with polymyalgia rheumatica two months ago, presented to the emergency department with anterior STEMI with left ventricle ejection fraction (LVEF) of $35 \%$ associated with akinesis of apex and hypokinesia of mid- to apical septal wall at transthoracic echocardiogram (TTE) (figure 1a, table 1, supplemental video 1a).

The patient had no coronary heart disease, no infections in the previous months and she was not allergic. Except for prednisone $7.5 \mathrm{mg}$, discontinued within the previous 36 hours and not referred at hospital admission, the patient was not taking any drugs.

After administering acetylsalicylic acid, ticagrelor and unfractionated heparin, by cannulating the right femoral artery, we performed percutaneous coronary intervention (PCI) placing a $12 \mathrm{~mm}$ length zotarolimuseluting stent on the proximal tract of the left anterior descending (LAD) (figures 2a and 2b; supplemental videos $2 \mathrm{a}$ and $2 \mathrm{~b}$ ), where a critical occlusion was found. At the end of the procedure thrombolysis in myocardial infarction (TIMI)-III flow was achieved (figure 2c; supplemental video 2c), so the patient was transferred to the intensive coronary care unit (ICCU) whit normal hemodynamics (table 1) and electrocardiogram (ECG) normalization (figure $1 b)$. 
Seven hours later the patient developed chest pain, hypotension, tachycardia and tachypnea (table 1). On evaluation she was confused and sweaty, but apyretic; a mild systolic murmur was audible and no signs of pulmonary congestion and access-site complications were detected. Lab exams are shown in table 1, while 12-lead ECG (figure 1c), TTE (supplemental video 1b) and lung ultrasound showed, respectively, sinus tachycardia, anterolateral ST-segment elevation (STE) with reciprocal depression in lead aVR, LVEF of $30 \%$ with apex and mid- to apical septal akinesis, small and collapsing inferior vena cava and no acute valves dysfunction, pericardial effusion and pleural B-lines. Meanwhile, we placed a central venous catheter, started crystalloids, norepinephrine and dobutamine and performed head, chest and abdomen computed tomography (CT) ruling out iatrogenic aortic dissection (IAD), intracranial and other bleeding and suspected infectious foci. Later, after placing intra-aortic balloon pump (IABP), a new coronary angiography (CA) showed TIMI-III flow on the LAD, ruling out acute stent thrombosis (AST) and Kounis syndrome (KS) (figure 2d; supplemental video $2 \mathrm{~d}$ ).

Upon returned to ICCU, hemodynamics showed a mixed shock (table 1), so dobutamine was withdrawn and norepinephrine was titrated up to $3.0 \mathrm{mcg} / \mathrm{kg} / \mathrm{min}$. Moreover, after performing microbiological assays (table 1), we started intravenous vancomycin and piperacillin-tazobactam. Nonetheless, three hours later a refractory shock was still present. After sampling plasma cortisol and adrenocorticotropic hormone (ACTH), we administered intravenous hydrocortisone (100 mg bolus, followed by $8.3 \mathrm{mg} /$ hour) achieving hemodynamic stabilization (table 1) and regression of mental confusion within two hours and ECG normalization.

The following days hemodynamic remained within normal range, troponin, NT-proBNP and inflammatory markers decreased, LVEF gradually increased, all microbiological assays tested negative and cortisol and ACTH values were typical for adrenal insufficiency (10) (table 1). Hydrocortisone was tapered and switched by oral formulation, antibiotics had withdrawn, IABP was removed and norepinephrine was decreased and discontinued. On day seven the patient was discharged with LVEF of $45 \%$ (supplemental video 1c), conventional therapy for myocardial infarction, prednisone $25 \mathrm{mg}$ /day and scheduled for endocrinological re-evaluation. The final diagnosis was STEMI complicated by AC presenting as fTTS.

The central figure summarizes the case described above.

\section{DISCUSSION}

Main differential diagnosis of STE, myocardial injury (MI) and shock occurring few hours following STEMI treated with PCI without complications related to coronary intubation and target vessel or site intervention (3) encompass AST, IAD, TTS, acute bleeding, KS, septic shock and early post-acute myocardial infarction pericarditis. As the latter typically occurs within few days (5), we do not include it our discussion.

AST, a life-threatening condition whose mortality rate may reach $7 \%$, typically occurs within 24 hours after stent implantation and its diagnosis requires the fulfilment of the same criteria used for type 1 myocardial infarction and identification of a stent thrombus (11)(12). In our case, by performing a new CA, AST (and others coronary procedures complications) was ruled out.

IAD is a rare complication related to cardiac catheterization that may present with pericardial effusion, hemodynamic instability and STE and it is burdened by elevated mortality rate. Its prompt diagnosis is mandatory and typically is done during CA; alternatively it is achieved by performing echocardiography or CT scans (4). Our patient showed no images of dissection at the end of the first CA, moreover, by performing CT scan, IAD was excluded.

Acute bleeding and intracranial hemorrhage may be related to antithrombotic and anticoagulant agents. When occurring, the patient may show mental confusion and, in about $20 \%$ of cases, STE, MI and regional wall motion abnormalities (RWMA) sometimes resulting in hypotension and shock (12)(13). Nevertheless, by performing lab exams and CT scans, we ruled out any bleeding.

KS, a hypersensitivity coronary disorder, may be due to contrast medium injection. Generally, it shows allergic stigmates and may mimic STEMI leading to shock in about 3\% of cases. Three variants of KS are recognized: the first two are related to coronary spasm with or without atheromatous plaque erosion or 
rupture, while the third is due to coronary stent thrombosis (14). Our patient had no history of allergies, showed no allergic stigmates and CA showed TIMI-III flow, so KS was excluded.

Septic shock may occur after invasive cardiac procedures in which adequate sterility is not achieved and it is due to the host immune response to face the infection. Typically, it is a distributive shock responding to vasopressors and, when it occurs, the patient may show mental confusion, MI with or without STE and RWMA (mostly due to myocardial oxygen supply/demand imbalance) (12)(15). Our patient showed low inflammatory markers, absence of bacteria and viruses on microbiological assays, no suspected sources of infection at CT scans and at femoral access-site and no hemodynamic improvement despite fluids and high doses of vasopressors, so septic shock was ruled out.

TTS is due to excessive sympathetic stimulation and/or catecholamines excess induced to emotional and/or physical triggers, mainly affects postmenopausal women and may mimic STEMI sometimes leading to cardiogenic shock. It is characterized by RWMA without a significative coronary occlusion, at CA, responsible for the abnormality of kinesis observed. Based on RWMA, four major variants of TTS are recognized: apical, mid-ventricular, basal and focal (12)(6). As ECG and RWMA may mimic a localized STEMI, the latter form is often difficult to diagnosticate without CA. Nevertheless, fTTS shows some peculiarities that differentiate it from other forms: a less reduction of LVEF, RWMA and ECG abnormalities mainly localized to the mid-interventricular septum and the anterolateral LV wall and a good prognosis (16).

Having ruled out other causes of shock presenting with STE and MI, according to the current diagnostic criteria and literature data (6)(16), the most likely diagnosis was fTTS. Indeed, our patient showed anterior STE, mid- to apical septal wall hypokinesis with only mild LVEF reduction (35\% to 30\%) and no complications during recovery. Moreover, by combining clinical presentation, mixed shock, prompt symptoms resolution and hemodynamic improvement only after hydrocortisone infusion and cortisol and ACTH values, diagnosis of AC due to adrenal insufficiency was made (8)(10).

We postulated that both recent discontinuation of prednisone and STEMI itself leaded to adrenal insufficiency. The former by giving negative feedback on the hypothalamus-pituitary-adrenal axis with insufficient cortisol production, while the latter, similarly to what previously reported (17), by developing proinflammatory cytokines leading to vasodilatation and hypotension due, in turn, to the loss of synergistic action of cortisol with endogenous catecholamines in maintaining vascular tone (9). Regarding the onset of fTTS during AC, according to what previously reported (7), we postulated a toxic effect of catecholamines on the myocardium due to the insufficient cortisol values resulting in RWMA. Although occurrence of TTS during adrenal insufficiency have been already described (7), to the best of our knowledge, this is the first case reporting the onset of the focal type.

\section{CONCLUSION}

Main causes of shock presenting with STE after a successful PCI encompass AST, acute bleeding, KS, TTS and KS and septic shock. TTS may be triggered by AC, which, in turn, could be due to the STEMI itself (acting as a stressful event) and/or previous use of cortisone. Although current literature describes cases of apical TTS, for the first time, we report the onset of focal TTS during adrenal insufficiency.

\section{REFERENCES}

1. Ibanez B, James S, Agewall S, Antunes MJ, Bucciarelli-Ducci C, Bueno H, et al. 2017 ESC Guidelines for the management of acute myocardial infarction in patients presenting with ST-segment elevation: The Task Force for the management of acute myocardial infarction in patients presenting with ST-segment elevation of the European Society of Cardiology (ESC). Eur Heart J [Internet]. 2017 Aug 26;39(2):119-77. Available from: https://doi.org/10.1093/eurheartj/ehx393

2. Aggarwal B, Ellis SG, Lincoff AM, Kapadia SR, Cacchione J, Raymond RE, et al. Cause of death within 30 days of percutaneous coronary intervention in an era of mandatory outcome reporting. J Am Coll Cardiol. $2013 ; 62(5)$. 
3. Giannini F, Candilio L, Mitomo S, Ruparelia N, Chieffo A, Baldetti L, et al. A Practical Approach to the Management of Complications During Percutaneous Coronary Intervention. Vol. 11, JACC: Cardiovascular Interventions. 2018.

4. Erbel R, Aboyans V, Boileau C, Bossone E, Di Bartolomeo R, Eggebrecht H, et al. 2014 ESC guidelines on the diagnosis and treatment of aortic diseases. Vol. 35, European Heart Journal. 2014.

5. Imazio M, Negro A, Belli R, Beqaraj F, Forno D, Giammaria M, et al. Frequency and Prognostic Significance of Pericarditis Following Acute Myocardial Infarction Treated by Primary Percutaneous Coronary Intervention. Am J Cardiol. 2009;103(11).

6. Ghadri J-R, Wittstein IS, Prasad A, Sharkey S, Dote K, Akashi YJ, et al. International Expert Consensus Document on Takotsubo Syndrome (Part I): Clinical Characteristics, Diagnostic Criteria, and Pathophysiology. Eur Heart J. 2018 Jun 7;39(22):2032-46.

7. Gupta S, Goyal P, Idrees S, Aggarwal S, Bajaj D, Mattana J. Association of Endocrine Conditions With Takotsubo Cardiomyopathy: A Comprehensive Review. J Am Heart Assoc. 2018 Oct 2;7(19).

8. Husebye ES, Pearce SH, Krone NP, Kämpe O. Adrenal insufficiency. Lancet. 2021 Feb;397(10274):613-29.

9. Rushworth RL, Torpy DJ, Falhammar H. Adrenal Crisis. N Engl J Med. 2019 Aug 29;381(9).

10. Hamrahian AH, Fleseriu M. Evaluation and Management of Adrenal Insufficiency in Critically ill Patients: Disease State Review. Endocr Pract. 2017 Jun;23(6):716-25.

11. Dangas GD, Claessen BE, Mehran R, Brener S, Brodie BR, Dudek D, et al. Clinical outcomes following stent thrombosis occurring in-hospital versus out-of-hospital: Results from the HORIZONS-AMI (Harmonizing Outcomes with Revascularization and Stents in Acute Myocardial Infarction) trial. J Am Coll Cardiol. $2012 ; 59(20)$.

12. Thygesen K, Alpert JS, Jaffe AS, Chaitman BR, Bax JJ, Morrow DA, et al. Fourth universal definition of myocardial infarction (2018). Eur Heart J. 2019;40(3).

13. Pinnamaneni S, Aronow WS, Frishman WH. Neurocardiac Injury after Cerebral and Subarachnoid Hemorrhages. Vol. 25, Cardiology in Review. 2017.

14. Abdelghany M, Subedi R, Shah S, Kozman H. Kounis syndrome: A review article on epidemiology, diagnostic findings, management and complications of allergic acute coronary syndrome. Vol. 232, International Journal of Cardiology. 2017.

15. Evans L, Rhodes A, Alhazzani W, Antonelli M, Coopersmith CM, French C, et al. Surviving sepsis campaign: international guidelines for management of sepsis and septic shock 2021. Intensive Care Med. $2021 ; 47(11)$.

16. Tini G, Limite LR, Arcari L, Cacciotti L, Russo D, Sclafani M, et al. A systematic review on focal takotsubo syndrome: a not-so-small matter. Heart Failure Reviews. 2020.

17. Norasyikin AW, Norlela S, Rozita M, Masliza M, Shamsul AS, Nor Azmi K. Adrenal insufficiency in acute coronary syndrome. Singapore Med J. 2009 Oct;50(10):962-6.

\section{FIGURE LEGENDS}

Central Figure: graphicAL representation of the case.

Figure 1a . ECG at Emergency Department. Negative and symmetrical T waves in leads DI, DII, aVL, aFV and from V2 to V6 and ST-segment elevation of $2 \mathrm{~mm}$ in V3 and of $1 \mathrm{~mm}$ in V4 and V4 (red arrows).

Figure 1b. ECG normalization after PCI.

Figure 1c. ECG during shock. Sinus tachycardia with ST-segment elevation of $1 \mathrm{~mm}$ in leads DI and DII and of $3 \mathrm{~mm}$ in leads V2 and V3. ST-segment depression of $1 \mathrm{~mm}$ in lead aVR (red arrows). 
Figure 2a. Coronary angiography: occlusion of the first tract of the LAD (red arrow) (caudal angulation).

Figure 2b. Coronary angiography: occlusion of the first tract of the LAD (red arrow) (cranial angulation).

Figure 2c. Coronary angiography after PCI: TIMI-III flows of the LAD (red arrow) (caudal angulation).

Figure 2d. Coronary angiography during shock: no AST nor coronary vasospasm (caudal angulation).

\section{VIDEO LEGENDS}

Supplementary Video 1a. Transthoracic echocardiogram at Emergency Department during STEMI. Apical 4 chambers view. LVEF of $35 \%$ with akinesis of apex and hypokinesia of mid- to apical septal wall.

Supplementary Video 1b. Transthoracic echocardiogram during shock. Reverse apical 4 chamber and subcostal views. LVEF of $30 \%$ with apex and mid- to apical septal wall akinesis and small and collapsing inferior vena cava.

Supplementary Video 1c. Transthoracic echocardiogram at discharge. Apical 4 and 2 chambers views. LVEF of $45 \%$ with apical and mid- to septal wall hypokinesia.

Supplementary Video 2a . Coronary angiography with occlusion of the first tract of the LAD (caudal angulation).

Supplementary Video 2b. Coronary angiography with occlusion of the first tract of the LAD (cranial angulation).

Supplementary Video 2c . Coronary angiography after PCI with TIMI-III flow of the LAD (caudal angulation).

Supplementary Video 2d. Coronary angiography during shock. No procedural and stent-related complications (caudal angulation).

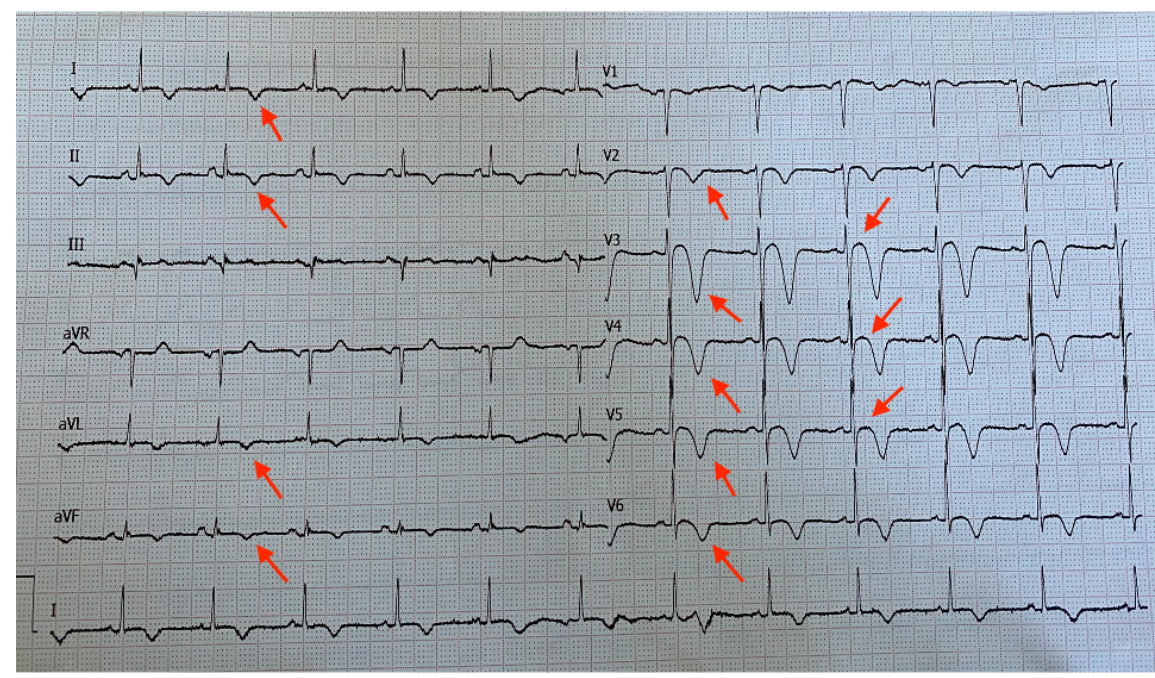



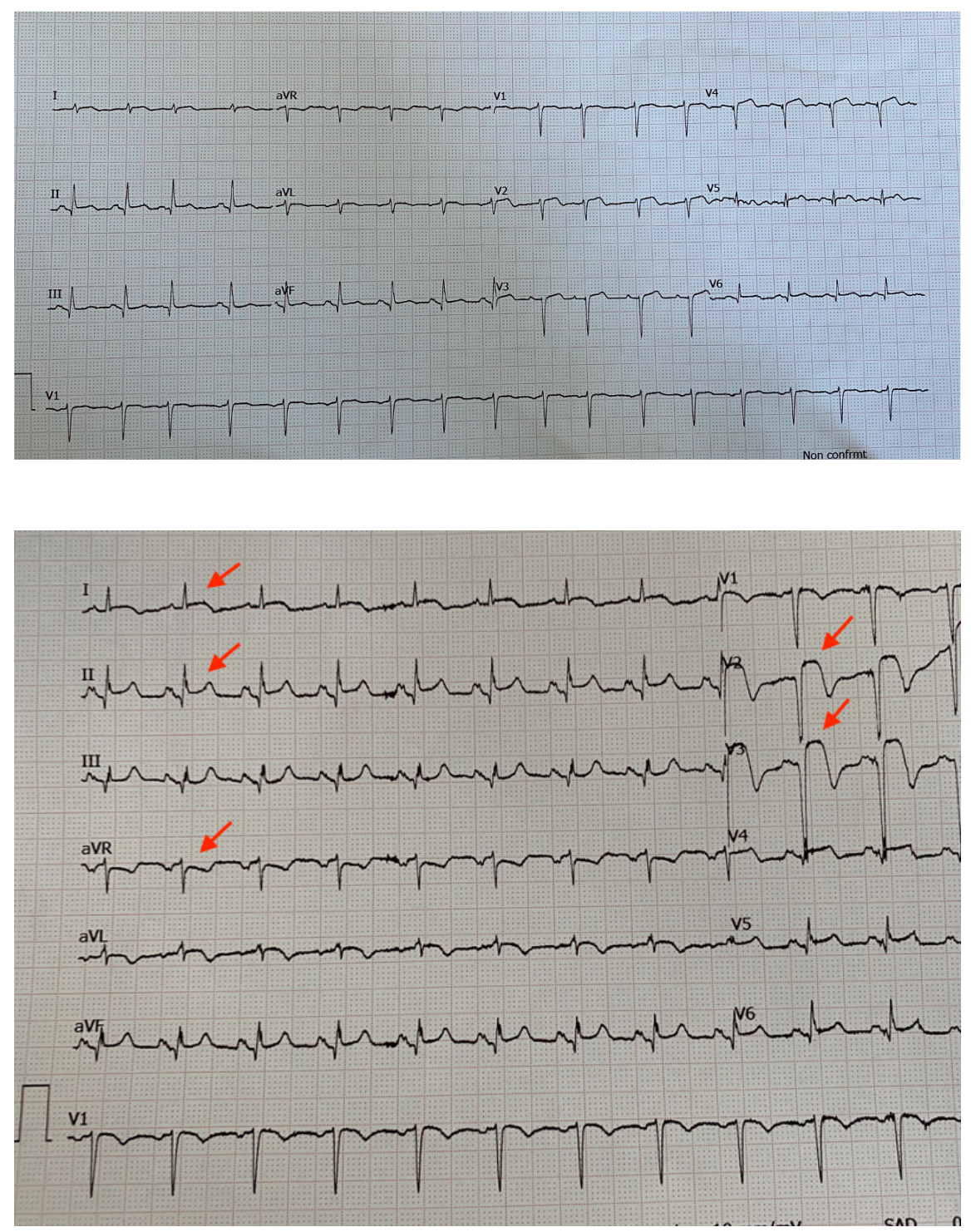


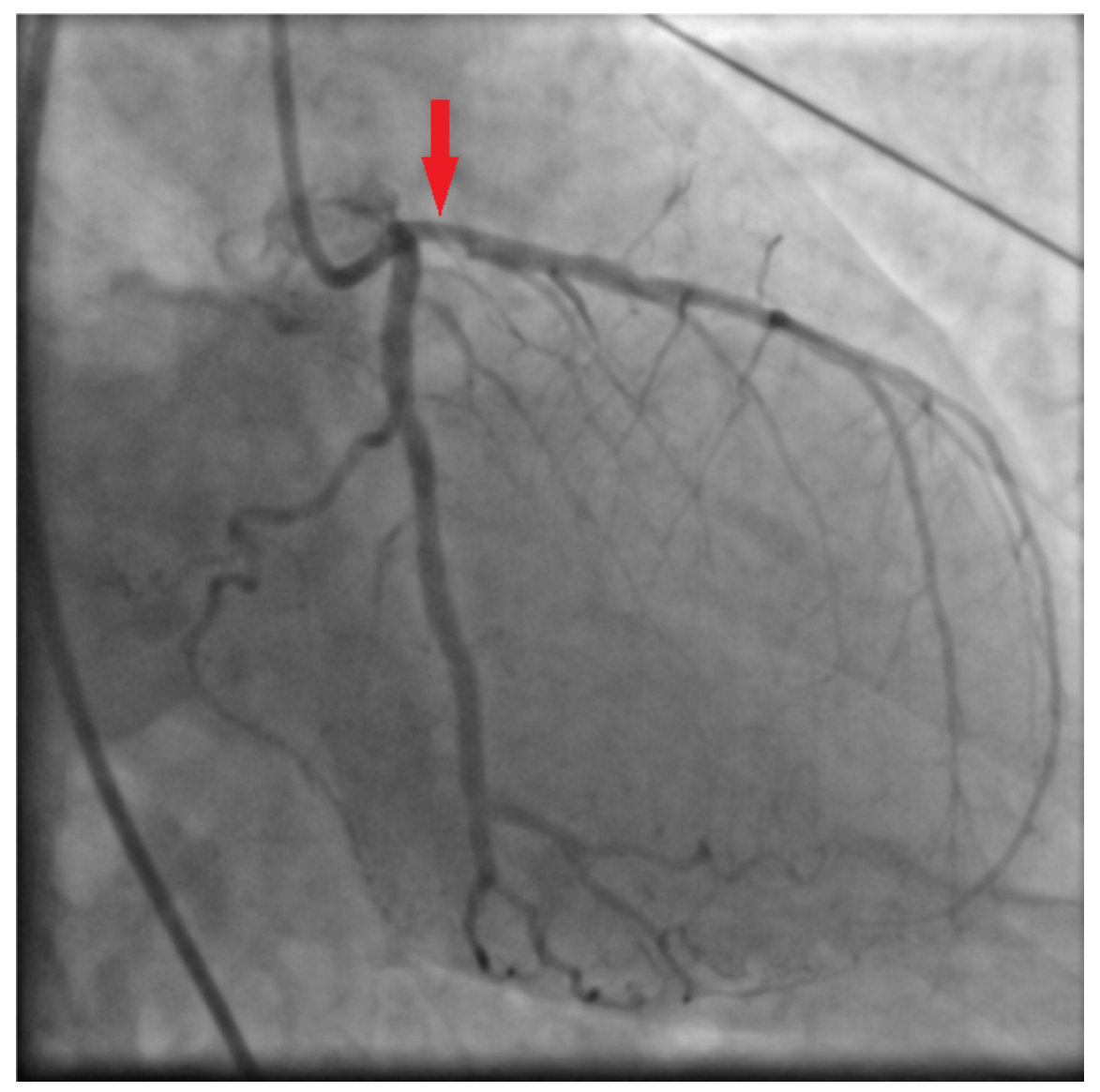




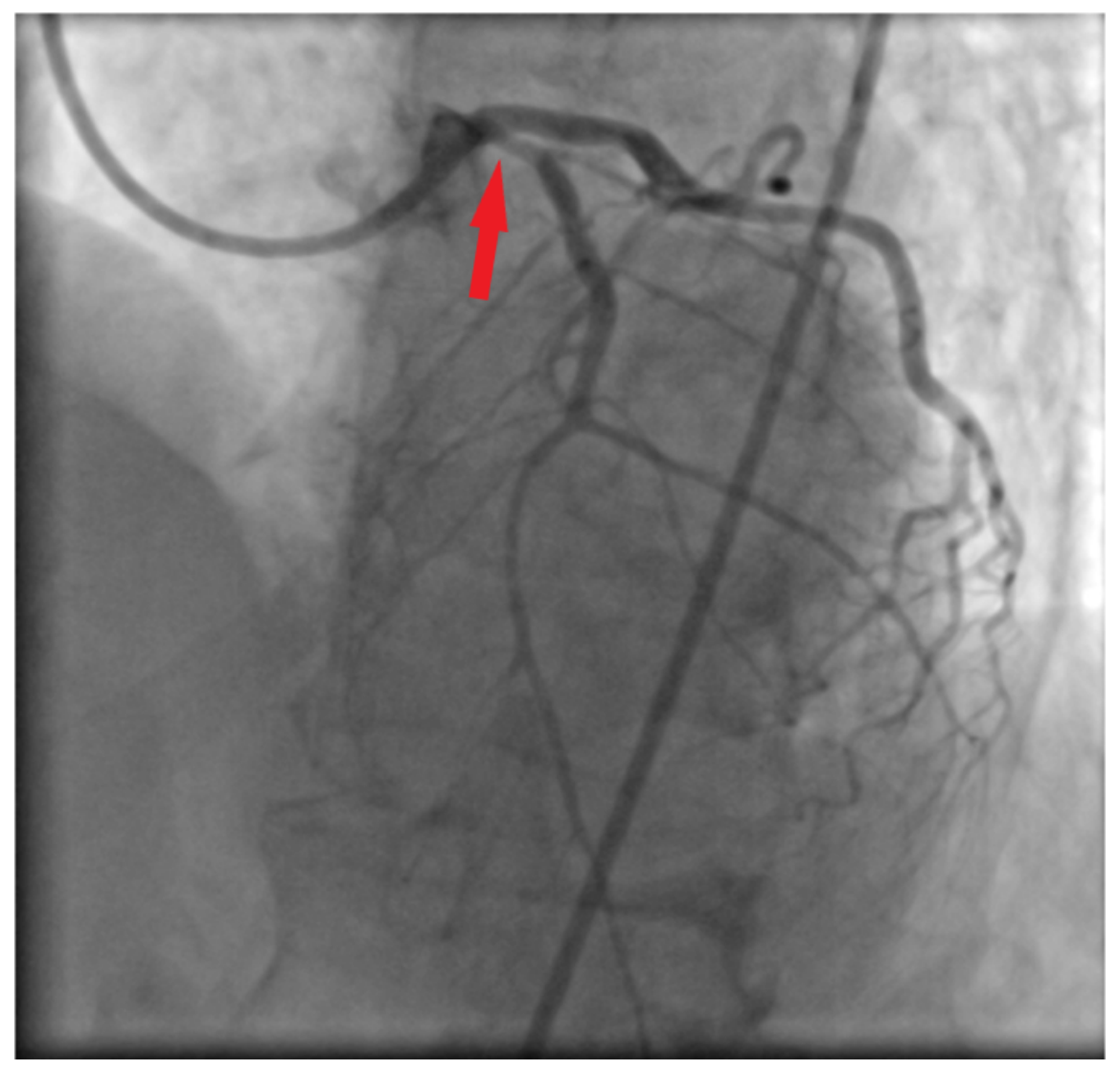




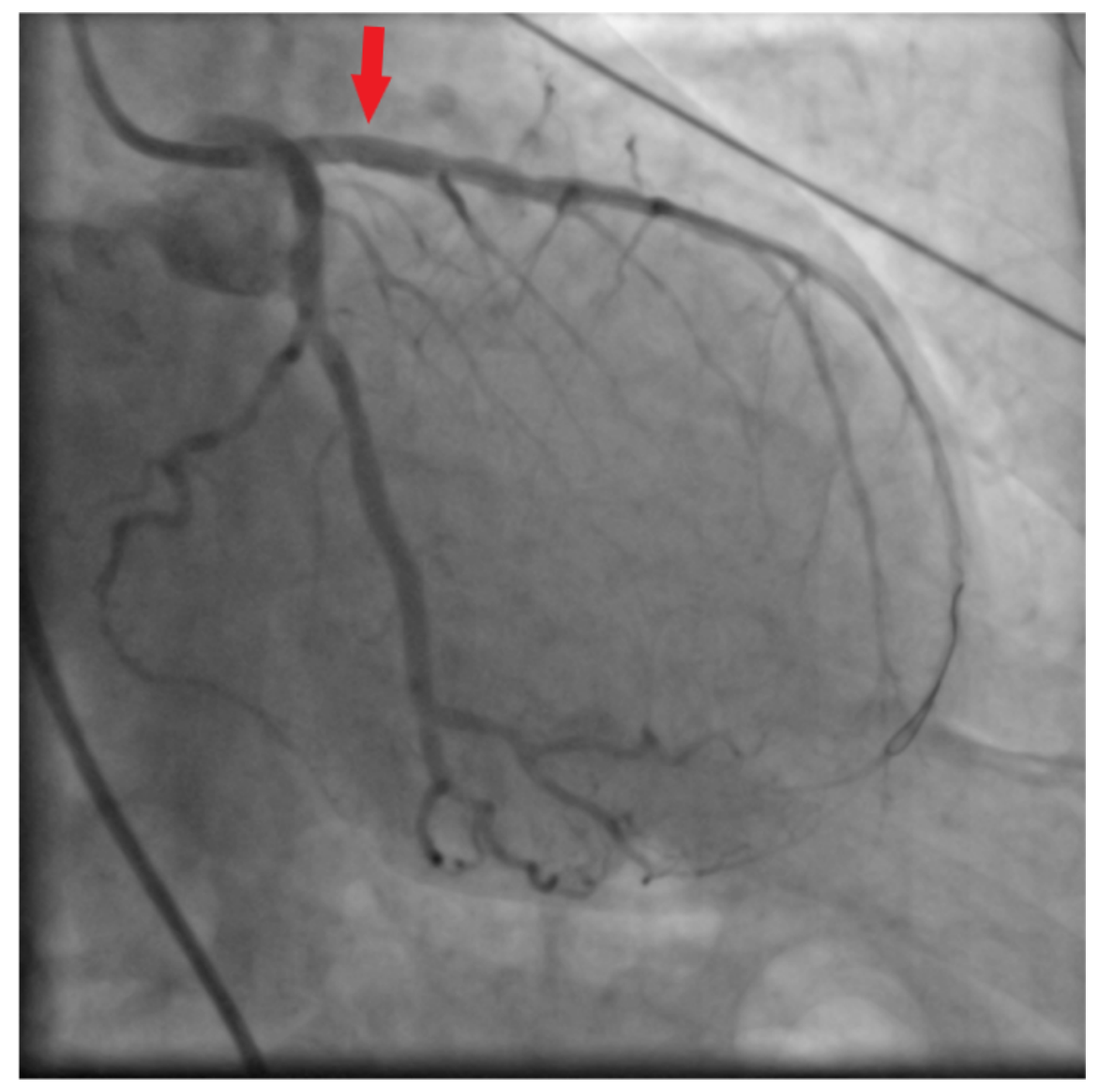




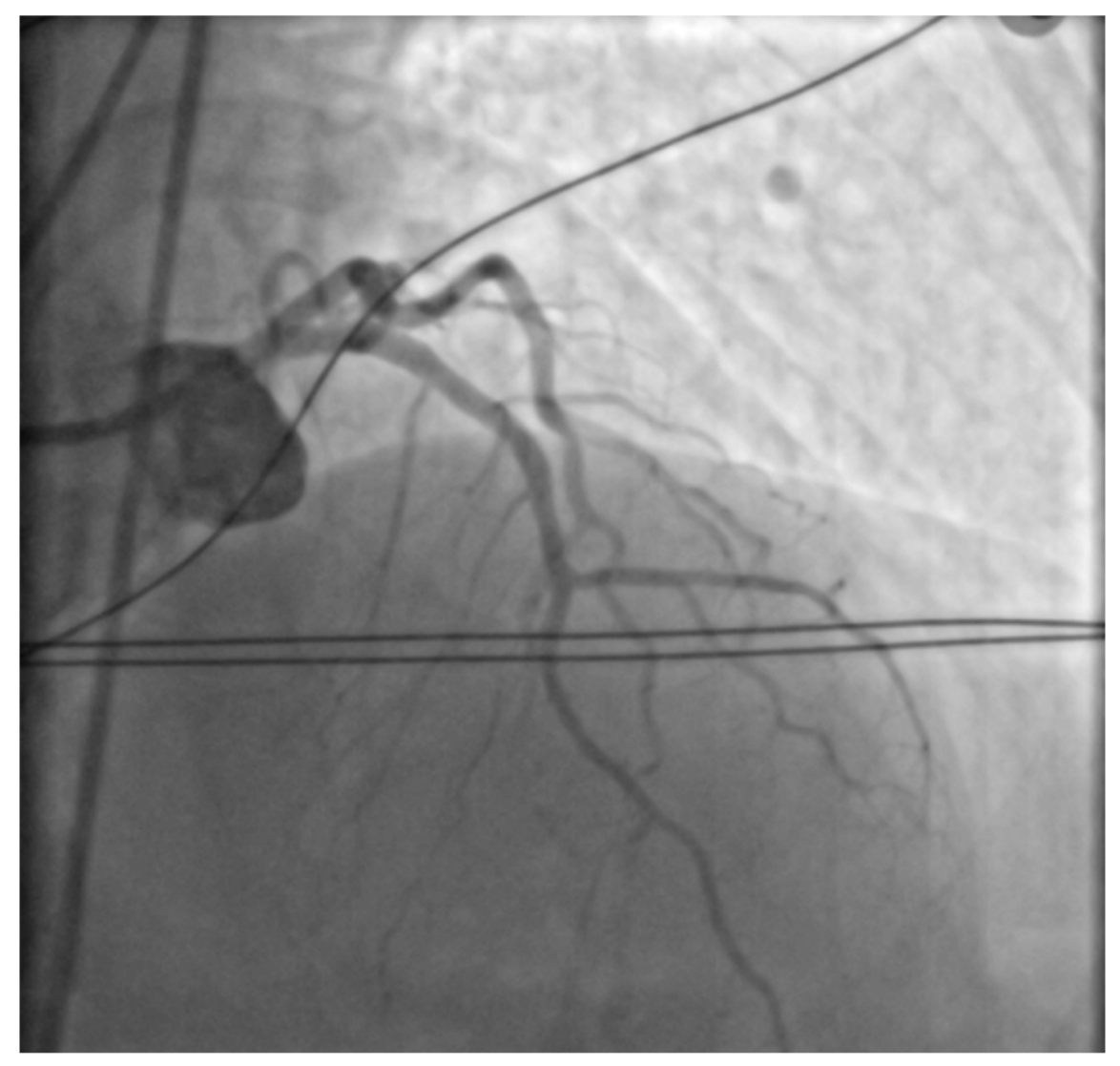

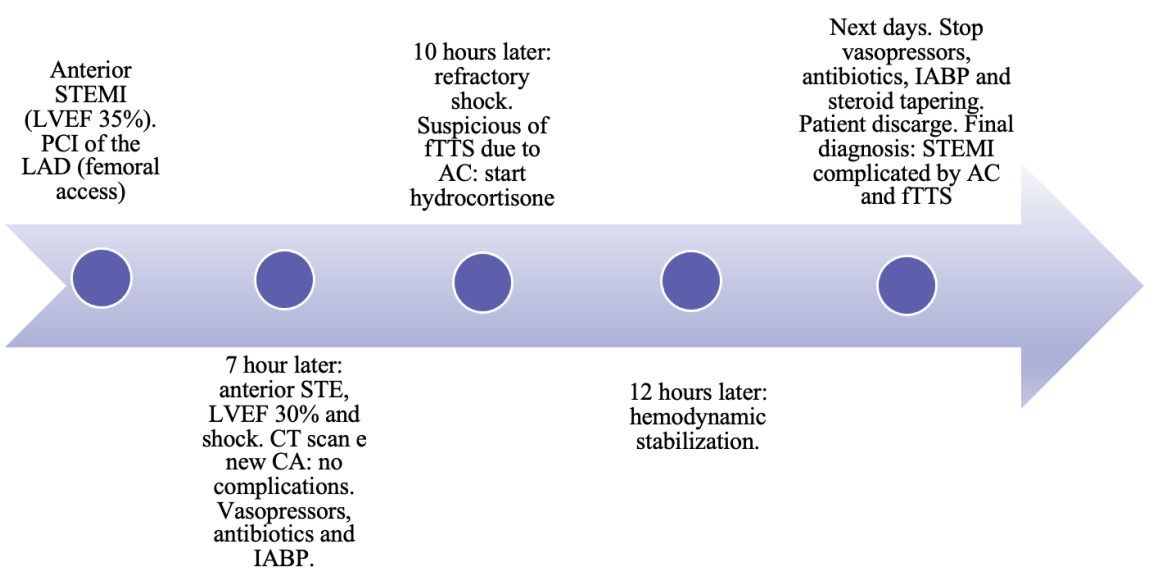

